\title{
Socioeconomic Inequalities in Gastroesophageal Reflux Disorder: Results from an Iranian Cohort Study
}

\author{
Mojtaba Farjam ${ }^{1}$, Mehdi Sharafi ${ }^{2}$, Ehsan Bahramali ${ }^{1}$, Satar Rezaei ${ }^{4}$, \\ Jafar Hassanzadeh ${ }^{3}$, Shahab Rezaeian ${ }^{4, *}$
}

1. Non-Communicable Diseases Research Center, Fasa University of Medical Sciences, Fasa, Iran

2. Center for Diseases Control and Prevention, Deputy of Health Services, Fasa University of Medical Sciences, Fasa, Iran

3. Research Centre for Health Sciences, Department of Epidemiology, School of Health, Shiraz University of Medical Sciences, Shiraz, Iran

4. Research Center for Environmental Determinants of Health, Kermanshah University of Medical Sciences, Kermanshah, Iran

\section{* Corresponding Author:}

Shahab Rezaeian, PhD Department of Epidemiology, School of Health, Kermanshah University of Medical Sciences, Isar Sq, Kermanshah 6719851351, Iran

Telefax: +988338262005

Email: shahab.rezayan@gmail.com

Received: 09 Feb. 2018

Accepted: 07 Jun. 2018

\section{ABSTRACT}

\section{BACKGROUND}

Despite progress in the health indexes in recent years, health inequalities remain as a global challenge within and between regions and countries. This study is the first to quantify the socioeconomic inequity in gastroesophageal reflux disease (GERD) using the concentration index.

\section{METHODS}

In this cross-sectional study, we used baseline data (7012 subjects) from the Fasa Cohort Study (the Southern Iran). The principal component analysis was used to construct socioeconomic status of the participants. The concentration index and concentration curve were used to measure socioeconomic-related inequality in GERD. Decomposition of concentration index was also done to identify the contribution of each explanatory variable to the wealth-related inequality in GERD prevalence.

\section{RESULTS}

The prevalence of GERD was $16.9 \%$ (95\% CI: $15.9-17.7 \%)$. The overall concentration index for GERD was 0.093 (95\% CI: 0.062-0.166]. Correspondingly, this figure for men and women were 0.116 (95\% CI: $0.062-0.171 \%$ ) and 0.091 (95\% CI: 0.044-0.137\%), respectively. The main contributors of socioeconomic-related inequality in GERD prevalence were socioeconomic status (64.4\%), alcohol drinking (29\%), and age (8.4\%).

\section{CONCLUSION}

GERD is significantly more concentrated among richest people. There was significant socioeconomic inequality in GERD according to some individual factors. These inequalities need to be addressed by policy makers to identify the vulnerable subgroups and to reduce the disease burden in the community.

\section{KEYWORDS:}

Gastroesophageal reflux, Concentration index, Inequality, PERSIAN Cohort, Iran

Please cite this paper as:

Farjam M, Sharafi M, Bahramali E, Rezaei S, Hassanzadeh J, Rezaeian S. Socioeconomic Inequalities in Gastroesophageal Reflux Disorder: Results from an Iranian Cohort Study. Middle East J Dig Dis 2018;10:180-187. doi: 10.15171/mejdd.2018.108.

\section{INTRODUCTION}

Gastroesophageal reflux disease (GERD) is a common gastrointestinal (GI) disorder with a high prevalence rate worldwide. It was described by the pioneering Iranian traditional physicians more than 10 centuries ago, ${ }^{1-3}$ and its clinical symptoms were defined in detail and recorded precisely. ${ }^{4}$ In modern medicine, consensus to determine the definitions of GERD has been regularly updating the data regarding the disease definition and epidemiological issues. ${ }^{5}$ GERD affects $10-25 \%$ of the western population ${ }^{6-8}$ and represents an 
increasing incidence trend in both developed and developing countries. ${ }^{910}$ Studies from Iran also report the same prevalence of GERD as western societies. ${ }^{11-13}$ In Iran, a high prevalence of the disease has been reported following an increasing incidence pattern. ${ }^{9,14}$

GERD has a considerable economic burden on the society ${ }^{15}$ and decreases the quality of life resulting in social burdens. ${ }^{16}$ Many population-based studies have also reported various factors associated with GERD including age, sex,${ }^{17}$ smoking, ${ }^{18}$ physical activity, ${ }^{18}$ educational level, ${ }^{19}$ obesity, ${ }^{10}$ neuropsychiatric disorders, ${ }^{20}$ residency place, ${ }^{21}$ and nutritional and dietary factors. ${ }^{18}$

Despite progress in the health indexes in recent years, health inequalities within and between regions and countries remain as a global challenge. In health economics, it is essential to determine inequalities in diseases and their risk factors to help policy makers to make policies and design better interventions to control morbidity and mortality. To our knowledge, no study has been conducted to determine the socioeconomic inequalities in GERD. Therefore, this study is the first to quantify the socioeconomic inequity in GERD using the concentration index.

\section{MATERIALS AND METHODS}

In this cross-sectional study, which was conducted in 2015, we used baseline data from the Fasa Cohort Study (the Southern Iran).

This cohort study, which is a part of the Prospective Epidemiological Research Studies in IrAN (PERSIAN) ${ }^{22}$ is a prospective population-based cohort to investigate risk factors of non-communicable diseases (NCDs) among individuals aged 35 to 70 years in the southern Iran. These individuals were invited by the rural health care workers (Behvarz) to participate in the cohort study and are followed up every six months.

There is a computer-based registration process for the cohort. Before registration, an informed consent is obtained from every participant. Data were collected through face-to-face interviews using electronic questionnaires on socio-demographic characteristics, health and disease status, lifestyle, physical activities, and behavioral factors. In addition, clinical examination and anthropometric measurements were done during the 15year study period. All the electronic questionnaires were designed to be smart, which can detect the inaccurate data. This issue can increase the accuracy and validity of the entered data. More details of the cohort study have been published before. ${ }^{23,24}$

In this inequality study, we used the baseline data of the cohort including age, sex, educational level, marital status, occupation status, smoking (no, yes), and alcohol consumption (no, yes) as independent variables.

The main outcome was GERD symptoms. GERD was defined based on the Montreal definition and classification of GERD. ${ }^{25}$ Patients were diagnosed as having GERD if they had the history of regurgitation over the last year and/or prior to the past year. The frequency of GERD symptoms was recorded as never, daily, 1-2 times/week, 3-6 times/week, and 1-3 times/month. The frequencies were considered as dichotomous variable (no, yes) for analysis.

We used the household asset data to measure the socioeconomic status (SES) of the individuals. The asset data are commonly used in the developing countries such as Iran where there are difficulties to obtain the comprehensive information of household income and expenditure instead of using traditional money metric measures. In this study, the asset data included ownership or renting of residential home, the area of house, number of rooms, bathroom, refrigerator, microwave, freezer, washing machine, dishwasher, vacuum cleaner, computer, laptop, television (black and white, color, LED, or LCD), mobile phone, motorcycle, car or truck, and internet access at home.

The principal component analysis (PCA) was used to generate asset index as a measure of SES in the study. The asset index was categorized into five quintiles and each quintile included one fifth of the subjects. The first quintile consisted of the poorest people, while the fifth quintile included the richest ones.

The concentration index (CI) is commonly used to assess the socioeconomic-related inequalities in health and epidemiology. The $\mathrm{CI}$ is based on the concentration curves, which lies between -1 and +1 . A negative index reflects a higher distribution of outcome among poor people (the concentration line is above equality line) and a positive index reflects a higher distribution among rich ones (the concentration line is under equality line). The zero value considered as perfect equality. In this study, the CI was used based on Wagstaff approach. ${ }^{26}$ 
Table 1: Descriptive characteristics and the prevalence of GERD in Fasa city, Southern Iran, 2015

\begin{tabular}{|c|c|c|c|c|}
\hline \multirow{2}{*}{ Variables } & & \multirow{2}{*}{ N (\%) } & \multicolumn{2}{|c|}{ Prevalence of GERD } \\
\hline & & & Crude $(95 \%$ CI) & Age-adjusted ( $95 \% \mathrm{CI})$ \\
\hline \multirow[t]{2}{*}{ Age group } & $\leq 50$ years & $4000(57.1)$ & $17.9(16.8$ to 19.2$)$ & 17.1 (15.9 to 18.2$)$ \\
\hline & $>50$ years & $3012(42.9)$ & 15.4 (14.1 to 16.7$)$ & $14.5(11.3$ to 18.4$)$ \\
\hline \multirow[t]{2}{*}{ Sex } & Male & $3109(44.3)$ & $15.9(14.7$ to 17.2$)$ & 15.9 (13.6 to 18.6$)$ \\
\hline & Female & $3903(55.7)$ & $17.6(16.4$ to 18.8$)$ & 14.5 (12.6 to 16.7$)$ \\
\hline \multirow[t]{3}{*}{ Marital status } & Married & $6245(89.1)$ & $16.7(15.8$ to 17.7$)$ & 15.1 (12.7 to 17.9$)$ \\
\hline & Single & $233(3.3)$ & $14.2(10.2$ to 19.3$)$ & $17(14.4$ to 19.9$)$ \\
\hline & Other & $534(7.6)$ & 19.7 (16.5 to 23.3$)$ & $19.2(16.5$ to 22.2$)$ \\
\hline \multirow[t]{2}{*}{ Employment status } & Unemployment & $3596(51.30$ & $17.3(16.1$ to 18.6$)$ & $15.8(13.3$ to 18.7$)$ \\
\hline & Employment & $3416(48.7)$ & $16.4(15.2$ to 17.7$)$ & 16.4 (14.3 to 18.6$)$ \\
\hline \multirow[t]{2}{*}{ Obesity } & No & $5610(80)$ & $16.7(15.7$ to 17.7$)$ & 14.7 (12.6 to 17.1$)$ \\
\hline & Yes & $1402(20)$ & 17.7 (15.7 to 19.7$)$ & 16.1 (13.4 to 19.1$)$ \\
\hline \multirow[t]{2}{*}{ Smoking status } & No & $5180(73.9)$ & $16.7(15.7$ to 17.7$)$ & $12.6(11.1$ to 14.3$)$ \\
\hline & Yes & $1832(26.1)$ & $17.4(15.7$ to 19.2$)$ & $21.2(18.5$ to 24.3$)$ \\
\hline \multirow[t]{2}{*}{ Alcohol drinking } & No & $6574(93.7)$ & $16.6(15.7$ to 17.6$)$ & $15.2(12.9$ to 17.80 \\
\hline & Yes & $438(6.3)$ & $20(16.6$ to 24.1$)$ & 16.7 (13.4 to 20.1$)$ \\
\hline \multirow[t]{5}{*}{ Socioeconomic status } & Poorest & $1403(20)$ & $14.6(12.8$ to 16.6$)$ & $13.6(11.6$ to 15.8$)$ \\
\hline & Poorer & $1403(20)$ & $14.8(13.1$ to 16.8$)$ & $14.9(12.9$ to 17.1$)$ \\
\hline & Middle & $1403(20)$ & $16.2(14.3$ to 18.2$)$ & 16.3 (14.4 to 18.3$)$ \\
\hline & Richer & $1403(20)$ & $16.9(15.1$ to 19.10 & 17.7 (15.2 to 20.4$)$ \\
\hline & Richest & $1403(20)$ & 21.2 (19.6 to 23.9$)$ & 21.1 (18.6 to 23.8$)$ \\
\hline
\end{tabular}

95\% CI: 95\% Confidence interval, GERD: Gastroesophageal reflux disease

In addition, the decomposition of the CI was done to identify the contribution of each explanatory variable to the wealth-related inequality in GERD. ${ }^{27}$ A negative (positive) contribution reveals the wealth-related distribution of the factor. In other words, this means the relationship between the relevant factor and GERD contributes to a lower (higher) likelihood of GERD prevalence among the poor (rich) people. As stated, since dichotomous status (no, yes) of GERD was used in the analysis, the marginal effects obtained from a logit model was used in the decomposition analysis. All statistical analyses were performed by Stata software version 11 (StataCorp, College Station, TX, USA).

\section{RESULTS}

Data were available for 7012 subjects. The prevalence of GERD symptoms was reported among 16.9\% (95\% CI: $15.9-17.7 \%$ ) of the subjects. The mean age of the participants was 49.4 years $(\mathrm{SD}=9.6)$ and $55.7 \%$ were women. About $26.1 \%$ of the participants were smoker, $6.3 \%$ had reported alcohol drinking, and $48.7 \%$ of them were illiterate. The age-adjusted prevalence of GERD was $15.3 \%$ (95\% CI: 12.9 - 17.9\%). More details of demographic characteristics of respondents according to the variables by the GERD prevalence are shown in table 1 .

The results of normalized CI and concentration curve for men, women, and total samples are presented in table 2 and figure 1 . The normalized CI for whole sample was 0.093. This index for men and women were estimated to be 0.116 and 0.091 , respectively. These results illustrated that GERD is more concentrated among better-off people $(p<0.001)$. In addition, the concentration curve for men, women, and whole samples lie under the line of perfect equality showing that GERD was more prevalent among advantaged people.

Table 3 shows the contribution of each explanatory variable to the wealth-related inequality in the GERD prevalence. 
Table 2: Normalized concentration index in the prevalence of GERD

\begin{tabular}{lcccc}
\hline & Concentration index & P value & \multicolumn{2}{c}{ 95\% Confidence interval } \\
\cline { 3 - 5 } Male & 0.116 & $<0.001$ & Lower & 0.062 \\
\hline Female & 0.091 & $<0.001$ & 0.044 & 0.171 \\
\hline Total & 0.093 & $<0.001$ & 0.062 & 0.166 \\
\hline
\end{tabular}

GERD: Gastroesophageal reflux disease

Table 3: Decomposition results for socioeconomic inequality to the prevalence of gastroesophageal reflux disease

\begin{tabular}{|c|c|c|c|c|c|c|c|c|}
\hline & $\begin{array}{l}\text { Concentration } \\
\text { index }\end{array}$ & $\begin{array}{c}\text { Marginal } \\
\text { effects }\end{array}$ & Mean & Elasticity & $\begin{array}{l}\text { Concentration } \\
\text { index }\end{array}$ & Contribution & $\%$ Contribution & Total $\%$ \\
\hline \multirow[t]{2}{*}{ Age group } & $\leq 50$ years & Ref & 0.57 & Ref & Ref & Ref & Ref & \multirow{2}{*}{8.4} \\
\hline & $>50$ years & -0.020 & 0.43 & -0.051 & -0.153 & 0.008 & 8.4 & \\
\hline \multirow[t]{2}{*}{ Sex } & Male & Ref & 0.44 & Ref & Ref & Ref & Ref & \multirow{2}{*}{ c -13.7} \\
\hline & Female & a 0.037 & 0.56 & 0.123 & ${ }^{b}-0.104$ & -0.013 & -13.7 & \\
\hline \multirow[t]{3}{*}{ Marital status } & Married & Ref & 0.89 & Ref & Ref & Ref & Ref & \multirow{3}{*}{-5.9} \\
\hline & Single & -0.026 & 0.03 & -0.005 & -0.216 & 0.001 & 1.1 & \\
\hline & Other & 0.041 & 0.08 & 0.019 & -0.335 & -0.007 & -7 & \\
\hline \multirow{2}{*}{$\begin{array}{l}\text { Employment } \\
\text { status }\end{array}$} & Unemployment & Ref & 0.51 & Ref & Ref & Ref & Ref & \multirow{2}{*}{-0.2} \\
\hline & Employment & -0.001 & 0.49 & -0.003 & 0.074 & 0.000 & -0.2 & \\
\hline \multirow[t]{2}{*}{ Obesity } & No & Ref & 0.80 & Ref & Ref & Ref & Ref & \multirow{2}{*}{-0.2} \\
\hline & Yes & -0.004 & 0.20 & -0.005 & 0.047 & 0.000 & -0.2 & \\
\hline \multirow[t]{2}{*}{ Smoking status } & No & Ref & 0.74 & Ref & Ref & Ref & Ref & \multirow{2}{*}{1.6} \\
\hline & Yes & 0.026 & 0.26 & 0.040 & 0.038 & 0.002 & 1.6 & \\
\hline \multirow{2}{*}{$\begin{array}{l}\text { Alcohol drink- } \\
\text { ing }\end{array}$} & No & Ref & 0.94 & Ref & Ref & Ref & Ref & \multirow{2}{*}{29} \\
\hline & Yes & 0.026 & 0.60 & 0.097 & 0.278 & 0.027 & 29 & \\
\hline \multirow{5}{*}{$\begin{array}{l}\text { Socioeconomic } \\
\text { status }\end{array}$} & Poorest & Ref & 0.20 & Ref & Ref & Ref & Ref & \multirow{5}{*}{64.4} \\
\hline & Poorer & 0.004 & 0.20 & 0.005 & -0.399 & -0.002 & -2 & \\
\hline & Middle & 0.021 & 0.20 & 0.025 & 0.000 & 0.000 & 0 & \\
\hline & Richer & 0.028 & 0.20 & 0.033 & 0.401 & 0.013 & 14.3 & \\
\hline & Richest & 0.051 & 0.20 & 0.061 & 0.801 & 0.048 & 52.1 & \\
\hline Total observed & & & & & & 0.077 & & 83.3 \\
\hline Residual & & & & & & 0.016 & & 16.7 \\
\hline Total & & & & & & 0.093 & & 100 \\
\hline
\end{tabular}

${ }^{a}$ Compared with men, women have 3.7 percentage point higher probability of GERD prevalence. ${ }^{\mathrm{b}}$ The concentration index reveals that the proportion of women are more concentrated among the poor. ${ }^{\mathrm{c}}$ The total contribution percentage of socioeconomic-related inequality in GERD for each variable.


Fig.1: Concentration curve in the prevalence of gastroesophageal reflux disease among men, women, and total population based on their socioeconomic status 
Older age, being single, employment, and obesity were associated with lower probability of GERD prevalence (the negative and significant sign of marginal effects). Higher SES (household wealth), smoking, alcohol drinking, and female sex were associated with higher probability of GERD prevalence among the participants (the positive and significant sign of marginal effects).

Results of the CI for explanatory variables indicate that employment, obesity, smoking, alcohol drinking, and female sex were more concentrated among the rich, whereas older age and being single were concentrated among the poor (see table 3 ).

The total contribution percentage for each explanatory variable was also demonstrated in the last column of the table 3. The main contributors of socioeconomic-related inequality in GERD were socioeconomic status (64.4\%), alcohol drinking (29\%), and age (8.4\%). The results suggested that approximately $83.3 \%$ of socioeconomic-related inequality in GERD prevalence were explained by explanatory variables included in the study. The remaining $16.7 \%$ of inequality were not included in the analysis and other variables were responsible for this inequality.

\section{DISCUSSION}

The results of this study revealed the determinants of socioeconomic inequalities in GERD based on an Iranian cohort study. Although, there are several studies on the determinants of GERD symptoms worldwide, , $3,21,28,29_{\text {to }}$ our knowledge, this is the first study conducted regarding the evaluation of GERD inequalities.

Getting information about the prevalence of noncommunicable diseases is the first basic step to assess the effectiveness of preventive measures in all levels of disease prevention and modification. ${ }^{30}$ So our first focus was on obtaining epidemiological data. We found that $16.9 \%$ of the participants had GERD symptoms. Prevalence of GERD is estimated to be $10-20 \%$ in the Western countries but less than $6 \%$ in south and east Asia. ${ }^{31}$ Several epidemiological studies have been conducted in Iran and other countries to determine the prevalence of GERD, but with a remarkable diversity among the reports. This diversity is the result of difference in definitions, variability in genetics and moreover, diverse environmental factors including nutrition and diet. For example, the prevalence of GERD in Iranian general population is reported from a minimum of $2.8 \%,{ }^{32}$ to a maximum of $58.5 \% .{ }^{33}$ Based on the better methodology, in a large cohort study with 50,000 participants in the North of Iran a representative prevalence rate of $20 \%$ has been estimated. ${ }^{13}$ A systematic review has also reported the prevalence rate of $21.2 \%$ in Iran. ${ }^{15}$

The main finding of our study was quantifying the size of inequality in GERD in a general population. We found a significant inequality in the disease with normalized CI of 0.093. In other words, our results showed that GERD in the Iranian population was not only prevalent, but also concentrated in the richest population and of course was associated with some factors. As found in previous studies, the high prevalence of GERD is associated with overweight/obesity, ${ }^{13,34,35}$ dietary factors (fast food), ${ }^{36}$ lower educational level, ${ }^{37}$ and behavioral factors such as alcohol drinking, ${ }^{13,36}$ and smoking. ${ }^{13,35,38}$ On the other hand, there were more proportions of obesity, smoking status, and alcohol consumption in the higher SES.

We found inequality in GERD with regard to obesity, sex, age, job, marital status, smoking, and alcohol consumption. In addition, the decomposition analysis showed that wealth (64.4\%), alcohol drinking (29\%), and age (8.4\%) were the main contributors to the observed inequality in the GERD prevalence. Based on the studies conducted on income-related inequality in health, SES has been reported as the most important contribution of pro-rich inequality in health..$^{39,40}$ We found no similar study for comparison. But one, by Emamian and colleagues, ${ }^{41}$ reported that the concentration of the prevalence of risk factors of non-communicable diseases such as higher body mass index (BMI) was in the higher percentiles of economic rank of people. A meta-analysis, ${ }^{42}$ showed that obesity was clearly associated with low educational levels, being married, and female sex, which may explain this fact. Another study conducted in Thailand to examine the SES and emerging obesity revealed that improvement in SES was positively associated with obesity for men and inversely for women aged younger than 40 years. ${ }^{43}$ So obesity might be an important factor in the rich groups who suffer from GERD. However, it is reported that symptoms of GERD may occur regardless of BMI among Asian population. ${ }^{44}$

Another factor that might intervene in interpretation of the results of our study is Helicobacter pylori (H. pylori) 
infection. In Iranian population, the prevalence of $H$. pylori infection in healthy people is reported to be $30.6 \%$ to $82 \%{ }^{45} \mathrm{H}$. pylori infection in stomach can decrease acid production and subsequently reflux. ${ }^{46}$ On the other hand, $H$. pylori infection is reported to be more frequently seen in low socioeconomic state. ${ }^{47}$ As a consequent, low socioeconomic people might have more prevalence of H. pylori infection, which in turn, this infection might have protected them from GERD. So a further step in this study would be the evaluation of $H$. pylori infection in the GERD population of both high and low socioeconomic statuses in a comparative manner.

We had some limitations in this study. The baseline data from the cohort was cross-sectionally used, which only involved the people older than 35 years. Hence, our results need to be repeated on other age subgroups. In addition, the poor people may have the lower rate of referral to the physicians, which could have led to lower diagnosis rate of GERD. This issue may raise the information bias. In this regard, Mohammadbeigi and colleagues $^{48}$ showed the inequality in health care utilization. They reported that the referral rate of general physicians and specialists are more concentrated in the richest people. Despite its limitations, this study is the first to quantify the size of inequality in GERD by using the largest data from a cohort study.

\section{CONCLUSION}

GERD is significantly more concentrated among richest people. There was significant socioeconomic inequality in GERD according to some individual factors. These inequalities need to be addressed by policy makers to identify the vulnerable subgroups and to reduce the disease burden in the community.

\section{ACKNOWLEDGMENTS}

We appreciate the scientific comments of Dr. Seyyed Ala Kazemeini, the gastroenterologist of Fasa University of Medical Sciences. This study was approved and partly funded by Fasa University of Medical Sciences (project no. 9407). The authors would like to thank all staff working in the Fasa NCDs cohort study. The authors declare that there is no conflict of interests.
There is nothing to be declared.

\section{CONFLICT OF INTEREST}

The authors declare no conflict of interest related to this work.

\section{REFERENCES}

1. Shirzad M, Mosaddegh M, Minaii B, Nikbakht Nasrabadi A, Ahmadian-Attari MM. The relationship between heart and stomach in Iranian traditional medicine: a new concept in cardiovascular disease management. Int J Cardiol 2013;165:556-7. doi:10.1016/j.ijcard.2012.09.006.

2. Shirbeigi L, Halavati N, Abdi L, Aliasl J. Dietary and Medicinal Herbal Recommendation for Management of Primary Bile Reflux Gastritis in Traditional Persian Medicine. Iran J Public Health 2015;44:1166-8.

3. Nadim M, Farjam M. Qutb al-Din Shirazi (1236-1311), Persian polymath physician in the medieval period. J Med Biogr 2016;24:360-2. doi:10.1177/0967772013518165.

4. Zohalinezhad ME, Askariz A, Farjam M. Clinical Stories and Medical Histories Recorded by Rhazes (865-925), the Iranian-Islamic Physician in the Medieval Period. Acta Med Hist Adriat 2015;13 Suppl 2:77-86.

5. Vakil N, van Zanten SV, Kahrilas P, Dent J, Jones R, Globale K. [The Montreal definition and classification of gastroesophageal reflux disease: a global, evidencebased consensus paper]. Z Gastroenterol 2007;45:112540. doi:10.1055/s-2007-963633.

6. Reichardt LA, Aarden JJ, van Seben R, van der Schaaf M, Engelbert RH, Bosch JA, et al. Unravelling the potential mechanisms behind hospitalization-associated disability in older patients; the Hospital-Associated Disability and impact on daily Life (Hospital-ADL) cohort study protocol. BMC Geriatr 2016;16:59. doi:10.1186/s12877-016-0232-3.

7. Gallastegi M, Guxens M, Jimenez-Zabala A, Calvente I, Fernandez M, Birks L, et al. Characterisation of exposure to non-ionising electromagnetic fields in the Spanish INMA birth cohort: study protocol. BMC Public Health 2016;16:167. doi:10.1186/s12889-016-2825-3.

8. Zendarski N, Sciberras E, Mensah F, Hiscock H. A longitudinal study of risk and protective factors associated with successful transition to secondary school in youth with ADHD: prospective cohort study protocol. $B M C P e$ diatr 2016;16:20. doi:10.1186/s12887-016-0555-4.

9. Sepanlou S, Khademi H, Abdollahzadeh N, Noori F, Malekzadeh F, Malekzadeh R. Time Trends of Gastroesophageal Reflux Disease (GERD) and Peptic U1cer Disease (PUD) in Iran. Middle East J Digest Dis 2010;2:78-83.

10. Okimoto E, Ishimura N, Morito Y, Mikami H, Shimura $\mathrm{S}$, Uno $\mathrm{G}$, et al. Prevalence of gastroesophageal reflux disease in children, adults, and elderly in the same community. J Gastroenterol Hepatol 2015;30:1140-6. doi: 10.1111/jgh.12899.

11. Mostaghni A, Mehrabani D, Khademolhosseini F,

\section{ETHICAL APPROVAL}


Masoumi SJ, Moradi F, Zare N, et al. Prevalence and risk factors of gastroesophageal reflux disease in Qashqai migrating nomads, southern Iran. World J Gastroenterol 2009;15:961-5. doi:10.3748/wjg.15.961.

12. Vossoughinia H, Salari M, Mokhtari Amirmajdi E, Saadatnia H, Abedini S, Shariati A, et al. An epidemiological study of gastroesophageal reflux disease and related risk factors in urban population of mashhad, iran. Iran Red Crescent Med J 2014;16:e15832. doi:10.5812/ircmj.15832.

13. Islami F, Nasseri-Moghaddam S, Pourshams A, Poustchi H, Semnani S, Kamangar F, et al. Determinants of gastroesophageal reflux disease, including hookah smoking and opium use- a cross-sectional analysis of 50,000 individuals. PLoS One 2014;9:e89256. doi:10.1371/journal. pone. 0089256

14. Redmond NM, Davies R, Christensen H, Blair PS, Lovering AM, Leeming JP, et al. The TARGET cohort study protocol: a prospective primary care cohort study to derive and validate a clinical prediction rule to improve the targeting of antibiotics in children with respiratory tract illnesses. BMC Health Serv Res 2013;13:322. doi:10.1186/1472-6963-13-322.

15. Delavari A, Moradi G, Elahi E, Moradi-Lakeh M. Gastroesophageal reflux disease burden in Iran. Arch Iran Med 2015;18:85-8. doi:015182/AIM.005.

16. Masoumi SJ, Khademolhosseini F, Mehrabani D, Moradi F, Mostaghni AA, Zare N, et al. Correlation of quality of life with gastroesophageal reflux disease amongst Qashqai nomads in Iran. Arch Iran Med 2012;15:747-50. doi:0121512/AIM.005.

17. Fujiwara Y, Higuchi K, Watanabe Y, Shiba M, Watanabe $\mathrm{T}$, Tominaga $\mathrm{K}$, et al. Prevalence of gastroesophageal reflux disease and gastroesophageal reflux disease symptoms in Japan. J Gastroenterol Hepatol 2005;20:26-9. doi:10.1111/j.1440-1746.2004.03521.x.

18. Rubenstein JH, Chen JW. Epidemiology of gastroesophageal reflux disease. Gastroenterol Clin North Am 2014;43:1-14. doi:10.1016/j.gtc.2013.11.006.

19. Hallan A, Bomme M, Hveem K, Moller-Hansen J, NessJensen E. Risk factors on the development of new-onset gastroesophageal reflux symptoms. A population-based prospective cohort study: the HUNT study. Am J Gastroenterol 2015;110:393-400. doi:10.1038/ajg.2015.18.

20. Reese ND, Schiller GJ. High-Dose Cytarabine (HD araC) in the Treatment of Leukemias: a Review. Current Hematologic Malignancy Reports 2013;8:141-8. doi:10.1007/ s11899-013-0156-3.

21. Foroutan M, Norouzi A, Molaei M, Mirbagheri SA, Irvani $\mathrm{S}$, Sadeghi A, et al. Eosinophilic esophagitis in patients with refractory gastroesophageal reflux disease. Dig Dis Sci 2010;55:28-31. doi:10.1007/s10620-008-0706-z.

22. Eghtesad S, Mohammadi Z, Shayanrad A, Faramarzi E, Joukar F, Hamzeh B, et al. The PERSIAN Cohort: Providing the Evidence Needed for Healthcare Reform. Arch Iran Med 2017;20:691-5.
23. Farjam M, Bahrami H, Bahramali E, Jamshidi J, Askari A, Zakeri $\mathrm{H}$, et al. A cohort study protocol to analyze the predisposing factors to common chronic non-communicable diseases in rural areas: Fasa Cohort Study. BMC Public Health 2016;16:1090. doi:10.1186/s12889-0163760-z.

24. Poustchi H, Eghtesad S, Kamangar F, Etemadi A, Keshtkar AA, Hekmatdoost A, et al. Prospective Epidemiological Research Studies in Iran (The PERSIAN Cohort Study): Rationale, Objectives and Design. Am J Epidemiol 2018;187:647-655. doi:10.1093/aje/kwx314.

25. Vakil N, van Zanten SV, Kahrilas P, Dent J, Jones R, Global Consensus G. The Montreal definition and classification of gastroesophageal reflux disease: a global evidencebased consensus. Am J Gastroenterol 2006;101:1900-20. doi:10.1111/j.1572-0241.2006.00630.x.

26. Wagstaff A, Paci P, Van Doorslaer E. On the measurement of inequalities in health. Soc Sci Med 1991;33:54557. doi:10.1016/0277-9536(91)90212-U.

27. Wagstaff A, Van Doorslaer E, Watanabe N. On decomposing the causes of health sector inequalities with an application to malnutrition inequalities in Vietnam. $J$ Econom 2003;112:207-23. doi:10.1016/S0304-4076(02)00161-6.

28. Asl SF, Mansour-Ghanaei F, Samadi H, Joukar F. Evaluations of life style factors and the severity of Gastroesophageal reflux disease; a case-control study. Int J Mol Epidemiol Genet 2015;6:27-32.

29. Khan A, Kim A, Sanossian C, Francois F. Impact of obesity treatment on gastroesophageal reflux disease. World J Gastroenterol 2016;22:1627-38. doi:10.3748/wjg.v22.i4.1627.

30. Fakhraei B, Farjam M. Evidence- Based Hypotheses about Levels of Prevention of Multiple Sclerosis: Current Findings, Future Prospects. La Prensa Medica Argentina 2014;100:3. doi:10.4172/lpma.1000130.

31. Wong BC, Kinoshita Y. Systematic review on epidemiology of gastroesophageal reflux disease in Asia. Clin Gastroenterol Hepatol 2006;4:398-407. doi:10.1016/j.cgh.2005.10.011.

32. Moghimi-Dehkordi B, Vahedi M, Khoshkrood Mansoori B, Kasaeian A, Safaee A, Habibi M, et al. Economic burden of gastro-oesophageal reflux disease and dyspepsia: A community-based study. Arab J Gastroenterol 2011;12:86-9. doi:10.1016/j.ajg.2011.03.005.

33. Khodamoradi Z, Gandomkar A, Poustchi H, Salehi AR, Imanieh MH, Etemadi A, et al. Prevalence and Correlates of Gastroesophageal Reflux Disease in Southern Iran: Pars Cohort Study. Middle East J Dig Dis 2017;9:12938. doi:10.15171/mejdd.2017.63.

34. Eslick GD. Gastrointestinal symptoms and obesity: a meta-analysis. Obes Rev 2012;13:469-79. doi:10.1111/ j.1467-789X.2011.00969.x.

35. Nasseri-Moghaddam S, Mofid A, Ghotbi MH, Razjouyan H, Nouraie M, Ramard AR, et al. Epidemiological study of gastro-oesophageal reflux disease: reflux in spouse as a risk factor. Aliment Pharmacol Ther 2008;28:144-53. doi:10.1111/j.1365-2036.2008.03708.x. 
36. Chirila I, Morariu ID, Barboi OB, Drug VL. The role of diet in the overlap between gastroesophageal reflux disease and functional dyspepsia. Turk $J$ Gastroenterol 2016;27:73-80. doi:10.5152/tjg.2015.150238.

37. Saberi-Firoozi M, Khademolhosseini F, Yousefi M, Mehrabani D, Zare N, Heydari ST. Risk factors of gastroesophageal reflux disease in Shiraz, southern Iran. World J Gastroenterol 2007;13:5486-91.

38. Nouraie M, Radmard AR, Zaer-Rezaii H, Razjouyan H, Nasseri-Moghaddam S, Malekzadeh R. Hygiene could affect GERD prevalence independently: a population-based study in Tehran. Am J Gastroenterol 2007;102:1353-60. doi:10.1111/j.1572-0241.2007.01208.x.

39. Ramezani Doroh V, Vahedi S, Arefnezhad M, Kavosi Z, Mohammadbeigi A. Decomposition of Health Inequality Determinants in Shiraz, South-west Iran. J Res Health Sci 2015; 15:152-8.

40. Rezaei S, Hajizadeh M, Khosravipour M, Khosravi F, Rezaeian S. Socioeconomic Inequalities in Poor Health-Related Quality of Life in Kermanshah, Western Iran: A Decomposition Analysis. J Res Health Sci 2018;18:e00405.

41. Emamian MH, Alami A, Fateh M. Socioeconomic Inequality in Non-Communicable Disease Risk Factors in Shahroud, Iran. Iran J Epidemiol 2011;7:44-51.

42. Djalalinia S, Peykari N, Qorbani M, Larijani B, Farzadfar F. Inequality of obesity and socioeconomic factors in Iran: a systematic review and meta- analyses. Med J Islam Repub Iran 2015;29:241.

43. Seubsman SA, Lim LL, Banwell C, Sripaiboonkit N, Kelly M, Bain C, et al. Socioeconomic status, sex, and obesity in a large national cohort of 15-87-year-old open university students in Thailand. J Epidemiol 2010;20:1320. doi:10.2188/jea.JE20090014.

44. Solhpour A, Pourhoseingholi MA, Soltani F, Zarghi A, Habibi M, Ghafarnejad F, et al. Gastro-esophageal reflux symptoms and body mass index: no relation among the Iranian population. Indian J Gastroenterol 2008;27:153-5.

45. Eshraghian A. Epidemiology of Helicobacter pylori infection among the healthy population in Iran and countries of the Eastern Mediterranean Region: a systematic review of prevalence and risk factors. World J Gastroenterol 2014;20:17618-25. doi:10.3748/wjg.v20.i46.17618.

46. Gisbert JP, Pajares JM, Losa C. Helicobacter pylori and gastroesophageal reflux disease: friends or foes? Hepatogastroenterology 1999;46:1023-9.

47. Wu JC. Does Helicobacter pylori infection protect against esophageal diseases in Asia? Indian J Gastroenterol 2011;30:149-53. doi:10.1007/s12664-011-0124-6.

48. Mohammadbeigi A, Hassanzadeh J, Eshrati B, Rezaianzadeh A. Socioeconomic inequity in health care utilization, Iran. J Epidemiol Glob Health 2013;3:139-46. doi:10.1016/j.jegh.2013.03.006. 